\title{
The association of attempted suicide with genetic variants in the SLC6A4 and TPHgenes depends on the definition of suicidal behavior: a systematic review and meta-analysis
}

\author{
RC Clayden ${ }^{1,2}$, A Zaruk ${ }^{2}$, D Meyre ${ }^{3}$, L Thabane ${ }^{1,2,3}$ and Z Samaan ${ }^{2,3,4}$
}

The global prevalence of suicide has increased substantially over the last four decades. Suicidal behavior manifests owing to a combination of biological, behavioral and social factors; however, the etiology of suicidality remains elusive. Even though twin studies have reported a significant heritability of $30-50 \%$, meta-analyses have not highlighted a common genetic variant associated with the spectrum of suicidal behavior. Here, we performed a systematic review of the literature $(n=112)$ to assess the association between serotonergic and non-serotonergic genetic polymorphisms and suicidal behavior. Using an inverse variance random-effects model, we developed pooled odds ratios for the 10 most commonly studied genetic variants related to suicidal behavior, each with at least five independent studies that met our stringent inclusion criteria. Our pooled results indicate no significant correlation between genetic polymorphisms and overall suicidal behavior. However, subgroups of suicide attempts demonstrated actual significance with the serotonin transporter (SLC6A4) 5HTTLPR (OR $=1.13$ (95\% confidence interval $=1.05-1.21), P=0.001)$ and reached nominal significance with the tryptophan hydroxylase rs1800532 (1.22 (1.05-1.41), $P=0.007$ ) variant. Subgroups of suicidal behavior (completions and attempts) displayed reduced heterogeneity compared with the overall suicidal behavior spectrum. Our findings suggest that the 5HTTLPR and rs 1800532 polymorphisms are significantly associated with suicide attempts, but not associated with completed suicides. The high degree of heterogeneity in past studies may be attributed to the lack of a phenotypic distinction between suicidal attempts and completions. Consequently, we have identified an important source of phenotypic heterogeneity that provides a rationale for the current lack of a common genetic variant associated with suicidal behavior.

Translational Psychiatry (2012) 2, e166; doi:10.1038/tp.2012.96; published online 2 October 2012

\section{Introduction}

Approximately one million people die by suicide each year and it is estimated that there are ten to twenty times as many suicide attempts. ${ }^{1}$ Suicidal behavior is a complex health and social issue that is believed to manifest as a combination of state-dependent factors, such as comorbid psychiatric and medical illnesses, and trait-dependent factors, including psychological, biological and genetic markers. ${ }^{2}$ In many communities, personal killing has long been embedded in culture and religion, though in the nineteenth century selfinjurious acts became widely recognized as an important medical issue. ${ }^{3}$ When making a clinical diagnosis, the World Health Organization describes suicide as an act of deliberate killing; however, a majority of the scientific community refers to suicidal behavior as the self-directed injurious acts with some intent to end one's own life. ${ }^{1}$ This working definition is far more inclusive of those individuals who exhibit thoughts of suicide and undertake suicidal attempts. Suicidal behavior can therefore be conceptualized on a phenotypic continuum ranging from suicidal ideation to suicidal attempt and completed suicide.

The trait-dependent heritable factors pertaining to suicidal pathology remain complex. Previous studies have demonstrated familial clustering of suicide and suicidal behavior, where the relative risk increased fivefold for relatives of suicidal individuals. ${ }^{4,5}$ Furthermore, in a twin case study, Voracek et al. found a significantly higher concordance rate $\left(P=4.1 \times 10^{-8}\right)$ for suicidal behavior in monozygotic $(24.1 \%)$ versus dizygotic $(2.8 \%)$ twins, establishing a significant genetic link. ${ }^{6}$ More recently, a genome-wide association study for suicide attempt identified a common variant (rs300774) at the ACP1 locus associated at a close to genome-wide level of significance $\left(P=5.07 \times 10^{-8}\right)$ with attempted suicidal behavior. ${ }^{7}$ However, as with many meta-analyses pertaining to suicidal behavior, GWA studies of suicide are limited by their inability to recognize complex phenotypic heterogeneity and distinct combinations of rare variants and environmental factors that may contribute to an increased risk of suicidal behavior. ${ }^{8}$

\footnotetext{
${ }^{1}$ Faculty of Health Sciences, McMaster University and the Father Sean O'Sullivan Research Centre, St. Joseph's Healthcare, Hamilton, ON, Canada; ${ }^{2}$ Population Health Research Institute, Hamilton, ON, Canada; ${ }^{3}$ Department of Clinical Epidemiology and Biostatistics, McMaster University, Hamilton, ON, Canada and ${ }^{4}$ Department of Psychiatry and Behavioral Neurosciences, McMaster University, Hamilton, ON, Canada

Correspondence: Dr Z Samaan, Department of Psychiatry and Behavioral Neurosciences, Mood Disorders Program, Centre for Mountain Health, St Joseph's Healthcare Hamilton, 100 West 5th, Hamilton, ON L8N 3K7, Canada.

E-mail: samaanz@mcmaster.ca
}

Keywords: meta-analysis; serotonin transporter; suicidal behavior; tryptophan hydroxylase

Received 20 April 2012; revised 23 August 2012; accepted 24 August 2012 
Suicide is an important subject owing to its worldwide prevalence, its burden on the health-care system and the severity of its social impact. Understanding and identifying the complexity of genes that may influence the progression of suicidality will help prevent future suicidal behavior and aid in designing more effective treatment strategies.

Neurobiological and genetic studies have suggested that suicidal behavior results from a complex interaction of several genes and stressful environmental factors. This hypothesis echoes a widely accepted stress-diathesis model among suicide literature, which presents suicidal attempts as a combination of stressors and abnormal familial psychological or physiological traits. ${ }^{1}$ Under this model, psychosocial or physiological pathologies may trigger suicidal ideation; however, it is the innate genotypic and phenotypic profile of the individual, which determines their inability to cope with stressors. Many specific genetic variants may exhibit an additive effect on suicidal behavior by intensifying the impact of a stressor. Genes involved in the serotonergic, adrenergic, noradrenergic and dopaminergic neurotransmitter systems have been studied in many samples of postmortem brain tissues, and many genetic variants have shown to vary neurotransmitter activity among suicide victims and controls. For our review, we performed an electronic search to identify studies that focused on the association between candidate genes and suicidal behavior.

With many genetic variants, the high degree of disagreement and variance among previous studies has presented conflicting results, which confer both harmful and protective effects. The aim of our study was to compile results and develop meta-analyses for common genetic variants, which influence suicidal behavior from studies published up to August 2011. By examining the results of our meta-analyses, we hoped to identify the candidate genes that are significantly associated with suicidal behavior.

Genetic markers and suicide. Genetic markers have long been implicated in association with suicidal behavior. A large number of molecular genetic studies have attempted to identify individual genes associated with suicide. However, these studies have not provided conclusive evidence of a common genetic variant associated with suicidal behavior. The majority of candidate genes have investigated a link involved in neurotransmitter processing, especially the serotonergic system, likely owing to their established role in emotion and behavior. A majority of these genetic studies contain small sample sizes, a large number of independent variables and inadequate phenotypic characterizations.

Several studies have investigated an association between suicide and common serotonergic gene polymorphisms (for reviews Brezo et al. $^{9}$ and Arango et $a l^{10}$ ). Many studies suggest that a reduction of serotonin uptake and enhanced serotonergic neurotransmission in suicides may be related to the commonly investigated short allele $(S)$ of the serotonin transporter (SLC6A4) insertion/deletion polymorphism. ${ }^{11}$ Studies pertaining to the short allele of SLC6A4 have shown a decreased protein expression of the transporter in the brain and platelets and conflicting results with regard to suicidal behavior. ${ }^{12}$ Research has suggested that multiple serotonin system-related genes, including the SLC6A4, 5-HT1A receptor (5HTR1A), 5-HT1B receptor (5HTR1B) and 5-HT2A receptor (5HTR2A), may be involved in the development of major depression and the exacerbation of suicidal behavior. ${ }^{13}$ In addition, genes encoding enzymes involved in the production and metabolism of serotonin have strong implications in suicide research. Both tryptophan hydroxylase $(T P H)$, the rate-limiting enzyme in the synthesis of serotonin, and monoamine oxidase $\mathrm{A}(M A O A)$ have been implicated in impulsivity, aggression and suicidal behavior. ${ }^{14,15}$

In addition, several non-serotonergic candidate genes have been potentially associated with suicide based on their function and role in the pathophysiology of emotion and behavior. ${ }^{9}$ Catechol-O-methyltransferase (COMT) is a major enzyme in catecholamine inactivation, which has been shown to influence aggressive and anger-related traits. ${ }^{16}$ Also, brain-derived neurotropic factor ( $B D N F)$ has been implicated in neuronal survival and plasticity and thought to be involved in various psychiatric disorders, attempted and completed suicide. ${ }^{17}$ Finally, imbalances in dopamine signaling are thought to have a role in the development of severe psychiatric disorders. The D2 receptor is known for its association with addictive behavior, while the D4 receptor has been associated with unipolar depressive disorder and obsessive compulsive disorder. ${ }^{18,19}$ Our research explored 35 different genes associated with suicidal behavior and multiple polymorphisms (single-nucleotide (SNP), variable number tandem repeats (VNTR) and insertions/deletions (indel)) characterized within each gene. With the wide spectrum of suicide-associated genetic polymorphisms, we attempted to identify significant genetic variants by carrying out a systematic review and meta-analysis following the MOOSE guidelines (reporting of meta-analyses of observational studies).

\section{Materials and methods}

Literature search. The literature search was conducted by selecting all studies on the PubMed, Embase, Psych Info and MEDLINE databases. First, we searched the keywords 'suicide' and 'genetic or gene' to compile a list of genetic markers associated with suicidal behavior. Subsequent search terms included a combination of the keywords 'suicide' and each individual genetic marker (see Supplementary Information S1). The references in each study were also reviewed through Google Scholar in order to gain any additional studies that were not elucidated in the primary literature search. All published studies between the years 1965 and 29 July 2011, which investigated a direct relationship between the genetic marker and suicide, were included for further analysis and subjected to subsequent inclusion criteria. In these publications, the following genes and gene variants were studied: 5HTR1A, 5HTR1B, 5HTR1D, 5HTR1E, 5HTR1F, 5HTR2A, 5HTR2C, 5HTR5A, 5HTR6, ABCG1, ADRA2A, APOE, BDNF, CCK, COMT, CRHR1, DCP1, DHCR7, DRD2, DRD4, ESR1, GABRA3, GS3KB, HMGCR, LDLR, LPL, MAOA, NTRK2, RGS2, SCN8A, SLC6A4, TPH, TH, VAMP4 and WFS1.

Inclusion and exclusion criteria. An adapted MOOSE 2008 flow diagram was used to help identify, screen and 


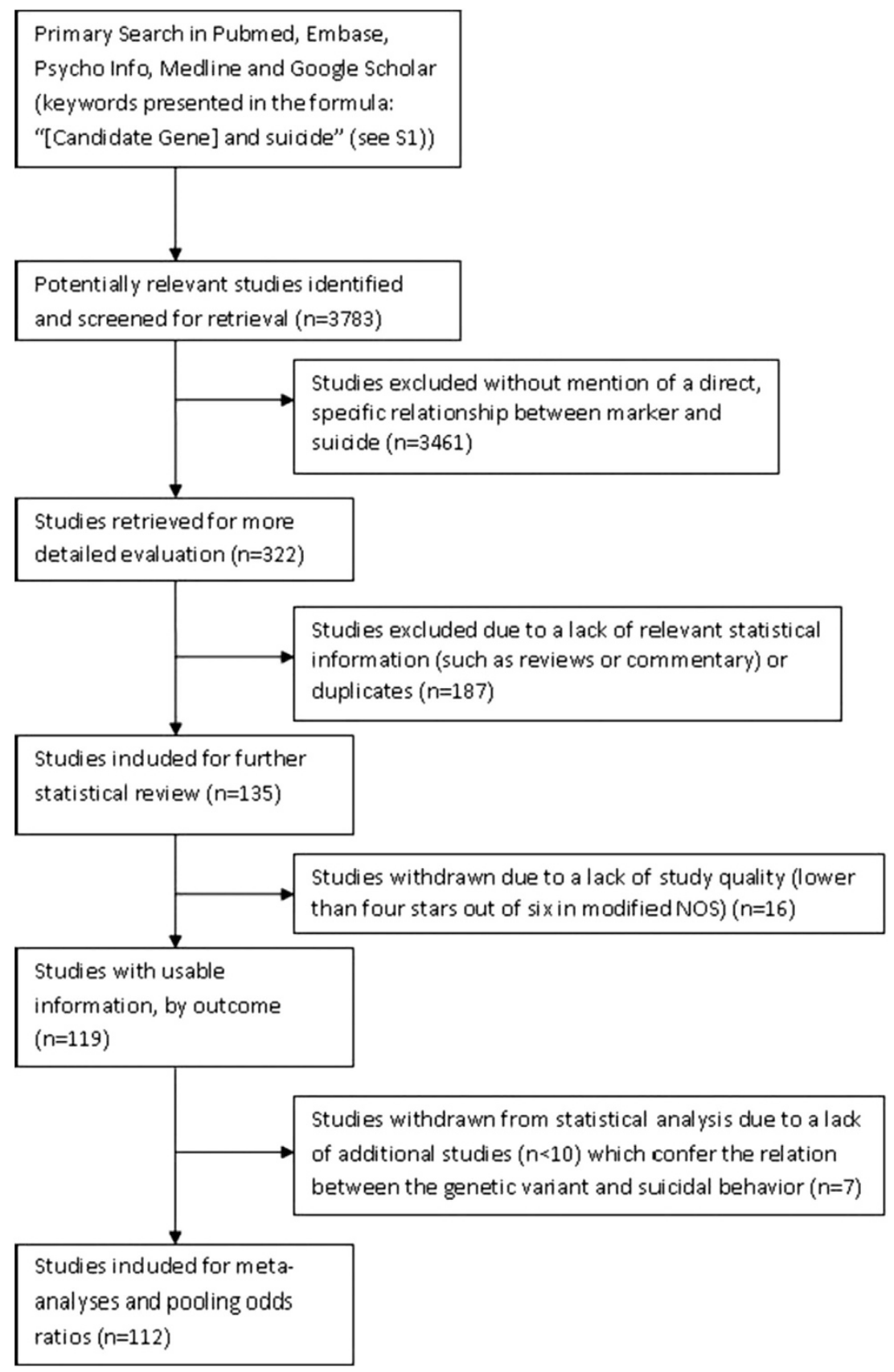

Figure 1 Methods for extraction and evaluation of pertinent genetic marker studies. We used a modified MOOSE flow chart to systematically evaluate the studies pertaining to the genetic markers associated with suicide. Each stage in the flow chart describes our inclusion criteria. From an initial screen of over 3000 studies, we found 112 high-quality case-controls for further statistical analysis.

select studies that were eligible for review and inclusion in our study (Figure 1). ${ }^{20}$ Studies that lacked the primary focus of a relationship between suicide and a specific genetic marker were excluded from the review (number of studies $(n)=3461)$. After a detailed evaluation of each study, studies that lacked pertinent statistical information for calculating allele frequencies or non-original studies (such as reviews, single case reports, editorials or commentaries) were excluded, resulting in 135 studies. Studies that investigated multiple alleles and, as a result, were present in multiple searches owing to our broad search terms $(n=52)$ were also removed owing to duplication. Data were extracted from primary studies by two independent reviewers (RCC and AZ; agreement $=98 \%$ ). In cases where there was a disagreement on the data extraction or inclusion criteria, a third author (ZS) was consulted to reach a consensus. Data extracted from primary studies included a definition of suicide (ideation/ attempt/completion), the mean age and sex of the cases and controls, comorbid psychiatric disorders, and the number of cases and controls. The type of sample population (general or clinical), each SNP investigated, and the minor allele frequencies were also recorded. 
Study quality. Each study $(n=135)$ was rated using a modified Newcastle-Ottawa Rating Scale, which examined the selection, comparability and exposure of each study based on six binary standards: case-control, community/hospital recruitment, definition of controls, mean age of recruitment (at least 18), definition of suicidal behavior and a method of ascertaining suicidal behavior (see Supplementary Information S2). Two independent authors (RCC and AZ) assessed each study for quality and where discrepancies existed they were resolved through discussion and consensus. We assessed the initial agreement using the Cohen kappa factor $(\kappa=0.87)$. Suicidal behavior was often classified by item 3 (suicidality) of the HAM-D-17 (Hamilton Depression Rating Scale, 17 items). ${ }^{13,21}$ Most studies defined suicide attempt as an intentional self-harm that required medical evaluation and treatment in an emergency or intensive care unit. Completed suicides were evaluated by the methods used. ${ }^{22}$ Lifetime diagnoses of psychiatric disorders were made according to DSM-IV criteria. ${ }^{23}$ Only high-quality case-control studies (at least four criteria met on the Newcastle-Ottawa Rating Scale) were used in the subsequent meta-analysis $(n=112)$. Corresponding authors were contacted if more data were required for inclusion in the analysis.

Meta-analysis. Our a priori null hypothesis was that a combination of studies assessing the association of genetic markers with suicide would not display a significant correlation with suicidal behavior. In addition, we undertook subgroup analyses separating attempted from completed suicides in order to explore possible phenotypic differences related to genetic associations. Subgroup analyses were applied to variants investigating suicide attempt or completion with at least 10 studies and a pooled sample size of at least 500 individuals owing to a lack of statistical power and small sample sizes. Statistical power of each sample group was calculated using the minor allele frequencies and sample sizes inputted into Quanto software (v1.2.4) (alpha $=0.05$, two-tail). ${ }^{24}$ After assessing the quality of each study, the minor allele frequencies of both the control and suicidal groups were recorded and entered into meta-analysis-generating software (Stata 12, StataCorp, College Station, TX, USA). Each study must have provided sufficient information to form $2 \times 2$ contingency tables and calculate an odds ratio (OR) within a 95\% confidence interval (Cl). Also, studies were analyzed for independent sample populations and excluded for duplicated results. Briefly, an inverse variance random effects DerSimonian and Laird model was used to calculate the effect of pooled ORs with the corresponding $95 \% \mathrm{Cl}$ and associated $P$ value. $^{25}$ By employing the non-iterative DerSimonian and Laird method, the authors accept that the studies included in the meta-analysis may vary in terms of the patient characteristics or study methods, and should not be weighted solely using sample sizes. A random-effects model was preferred over a fixed-effect model as a result of the high degree of interstudy variance observed in the preliminary results. Under this model, a combination of the mean effect of a population and deviation from the pooled effect, collectively known as the true treatment effect, was used with the s.e. to calculate a measure of the observed treatment effect. ${ }^{25}$ The inverse variance method was used to maximize the inclusiveness of studies in the meta-analyses (which reported limited statistical information). Nominal statistical significance was set a prioriat alpha $=0.05$, with a Bonferroni correction for multiple testing (revised alpha $=0.005$ ). The statistical heterogeneity (assessed by $I^{2}$ analysis) and funnel plots were used to explore the possibility of publication bias. It is often assumed that a lack of published studies with insignificant results or a lack of unpublished data in meta-analyses will create a noticeable publication bias. An asymmetrical effect on the funnel plot signifies potential publication bias or smaller study effects, which drive the pooled effect toward a falsely significant result.

\section{Results}

We reviewed 119 studies that investigated an association between serotonergic or non-serotonergic genes and suicidal behavior that met our selection and inclusion criteria (Figure 1). Twenty-seven of these studies examined the relationship between multiple genetic variants and suicide leading to a total investigation of 223 genetic variants. We chose the most common variants (analyzed in at least five studies passing the inclusion criteria) associated with suicidal behavior to calculate pooled ORs and investigate the significance of their findings. This reduced the final number of included studies to 112. In order to ensure high statistical power in subgroup analyses, we then generated forest plots for the variants with at least 10 studies (SLC6A4 (5HTTLPR), TPH (rs1800532), 5HTR2A (rs6313) and 5HTR1B (rs6296)). In addition to these four variants, we performed metaanalyses with six additional common genetic variants associated with suicide given their implicated roles in neurotransmission, psychopathology and suicidal behavior. As a result, 10 variants were investigated for pooled effects. The initial literature search was not restricted to studies written in English and we did not encounter any language barriers in our study as all of the included studies were written in or translated to English. The results of our findings are as follows:

The study designs were mainly case-control $(n=104)$ or nested case-controls $(n=8)$ with no cohort designs. The eligible studies for our review compared suicidal cases with control subjects and measured suicidal behavior as the main outcome. Contoured funnel plots were generated and analyzed using an Egger's plot to quantify the potential for bias in each meta-analysis. In some instances, the risk of publication bias in our meta-analyses was difficult to assess owing to the small number of studies, limited sample sizes and the differences in selected populations. Out of the 112 studies that we examined, 87 studies reported a known comorbid psychiatric disorders (including schizophrenia, depression, personality disorders and alcohol abuse), 22 studies had no mention of comorbidities, 2 studies stated unknown psychiatric disorders and 1 study had no psychiatric illnesses.

The Serotonin Transporter Gene (SLC6A4). As one of the most commonly studied genetic associations, our first analysis on the association between the serotonin transporter $\mathrm{S}$ allele and suicidal behavior contained 31 primary casecontrol studies (number of cases $(\mathrm{NS})=6324$; number of controls $(\mathrm{NC})=10285)$ with a pooled OR $(95 \% \mathrm{Cl})$ of 1.06 (0.90-1.26), $P=0.47$. When only the attempted suicide 


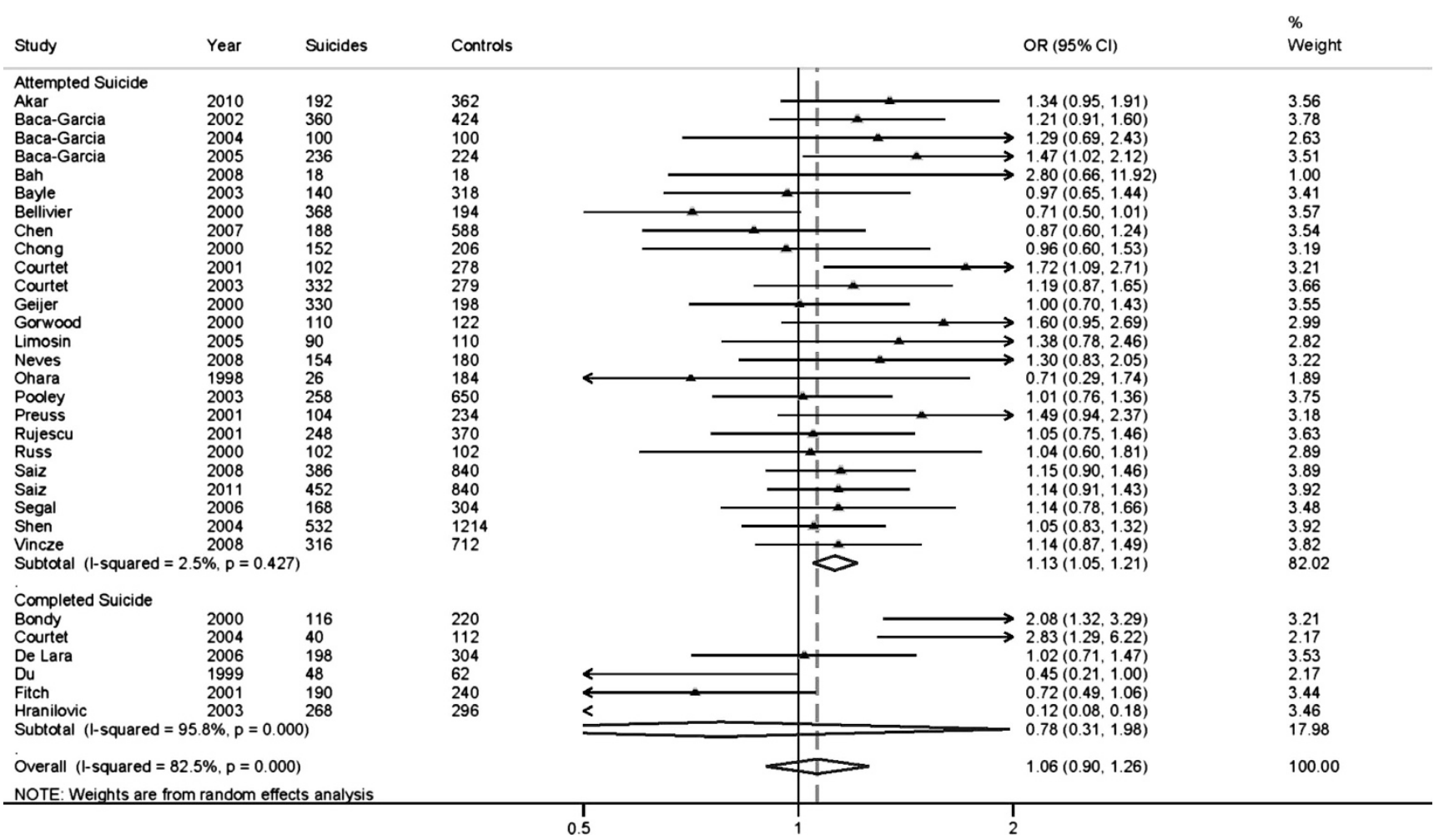

Figure 2 A forest plot for meta-analysis of the studies relating the serotonin transporter (SLC6A4) short polymorphism (5HTTLPR) to suicide attempts, completions and overall suicidal behavior. We applied a random-effects inverse variance model to pool the odds ratios associating the SLC6A4 short allele with suicidal behavior. This plot describes the results for the short allele relative to the long allele.

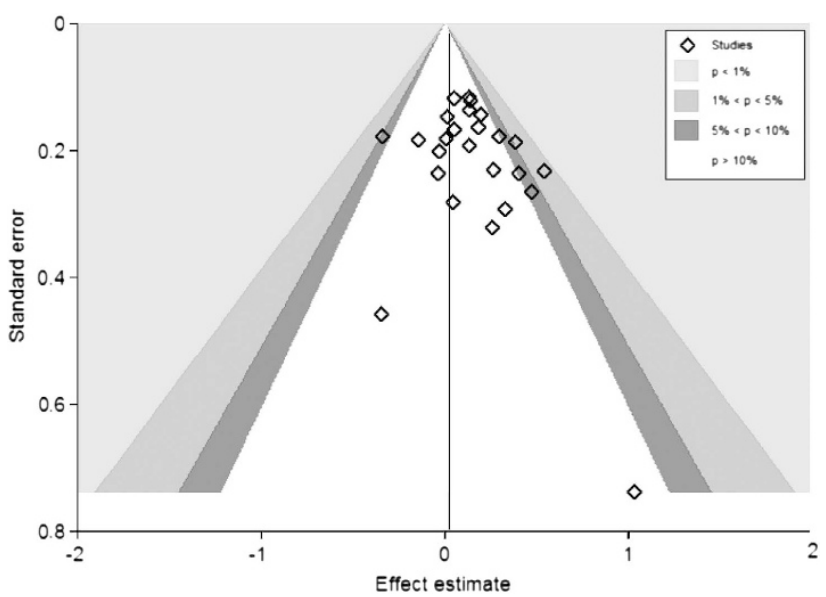

Figure 3 A funnel plot to assess publication bias of studies relating the SLC6A4 5 HTTLPR polymorphism to suicide attempts. We constructed contoured funnel plots to assess the risk of publication bias in our meta-analyses. This plot displays the outcome effect estimate in relation to the s.e. of the effect for each study included in our SLC6A4 meta-analysis. The figure shows a partially asymmetrical plot that signifies the possibility of publication bias.

case-control studies were analyzed $(n=25)$, the pooled OR reached actual significance at $1.13(1.05-1.21)(P=0.001$ and power of $99 \%)$ and the heterogeneity $\left(I^{2}=2.5 \%\right)$ decreased by $80 \%$ (Figure 2). The pooled completed suicide case-control studies $(n=6)$ were nonsignificant $(0.78(0.31-$ 1.98) with a power of $98 \%$ ) and had a high degree of heterogeneity $\left(I^{2}=95.8 \%\right)$. Approximately $81 \%$ of all studies involving the 5 HTTLPR polymorphism and suicidal behavior reported suicide attempts. A contoured funnel plot was generated, with the studies reporting attempted suicides and the SLC6A4 S allele, in order to assess the possibility of publication bias or small study effects (Figure 3 ). The s.e. was plotted against the estimated OR of each study and displayed a slightly asymmetrical pattern.

The serotonin receptor genes. The family of serotonin receptors (5HTR) contains several subtypes and isoforms that have inconsistently been linked to suicidal behavior through genetic associations. In this study, 4 of the 10 polymorphisms represented members of the 5HTR family (rs6313 (5HTR2A, $n=18, \mathrm{NS}=3759, \mathrm{NC}=5692$ ), rs6318 (5HTR2C, $n=7, \mathrm{NS}=2297, \mathrm{NC}=3431)$, rs6295 (5HTR1A, $n=6, \mathrm{NS}=2022, \mathrm{NC}=2135)$ and $\mathrm{rs} 6296$ (5HTR1B, $n=10$, $\mathrm{NS}=2947, \mathrm{NC}=4066)$ ). These four SNPs displayed nonsignificant pooled ORs of $0.92(0.81-1.04), P=0.11$, power of $80 \% ; 0.90(0.73-1.11), P=0.41$, power of $49 \% ; 1.14$ (0.89-1.47), $P=0.30$, power of $84 \%$; and 1.07 (0.85-1.35), $P=0.76$, power of $44 \%$, respectively. For the rs6313 and rs6296 variants, studies involving completed suicides were removed from the analyses and retested. The adjusted pooled ORs of attempt suicide for each genetic marker were nonsignificant $(n=13, \quad \mathrm{OR}=0.93 \quad(0.82-1.06), \quad P=0.28$, power of $61 \%, I^{2}=41 \%$ and $n=5$, OR $=0.90(0.75-1.09)$, $P=0.30$, power of $37 \%, I^{2}=0 \%$, respectively) as were the pooled ORs of completed suicides $(n=5$, OR $=0.85$ (0.651.12), $P=0.13$, power of $56 \%, I^{2}=44 \%$ and $n=5$, OR $=1.29(0.89-1.87), P=0.17$, power of $99 \%, I^{2}=84 \%$, respectively) (Figure 4, 5HTRIB rs6296). 


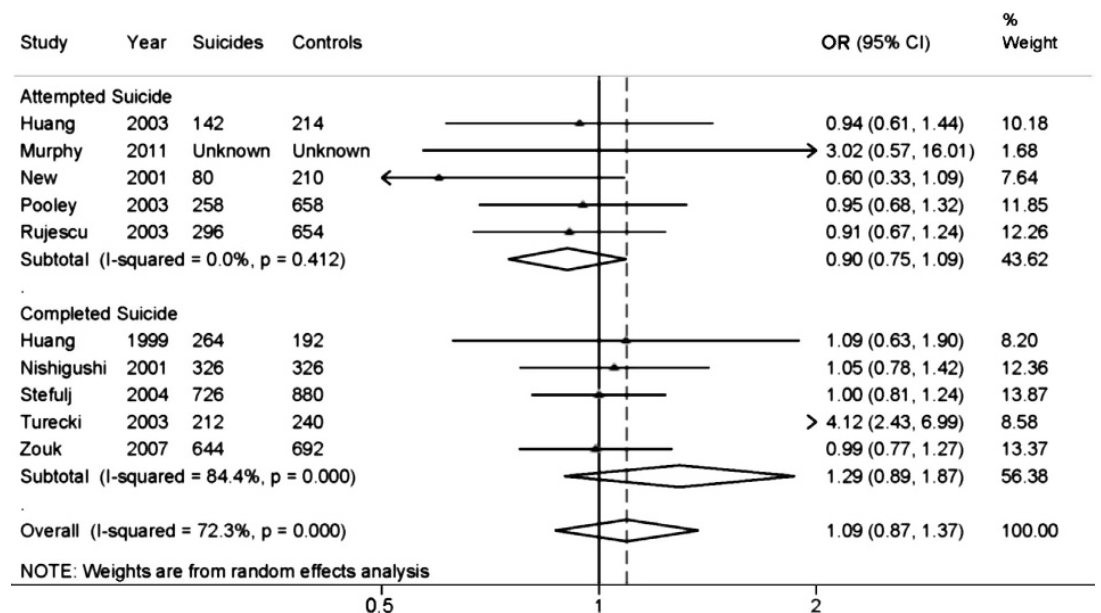

Figure 4 Forest plot for random-effects meta-analysis of the studies relating the $5 H T R 1 B$ rs 6296 variant to suicide attempts, completions and overall suicidal behavior. We applied a random-effects inverse variance model to pool the odds ratios associating the 5HTR1B minor allele with suicidal behavior. This plot describes the results for the G861 allele relative to the $\mathrm{C} 861$ allele.

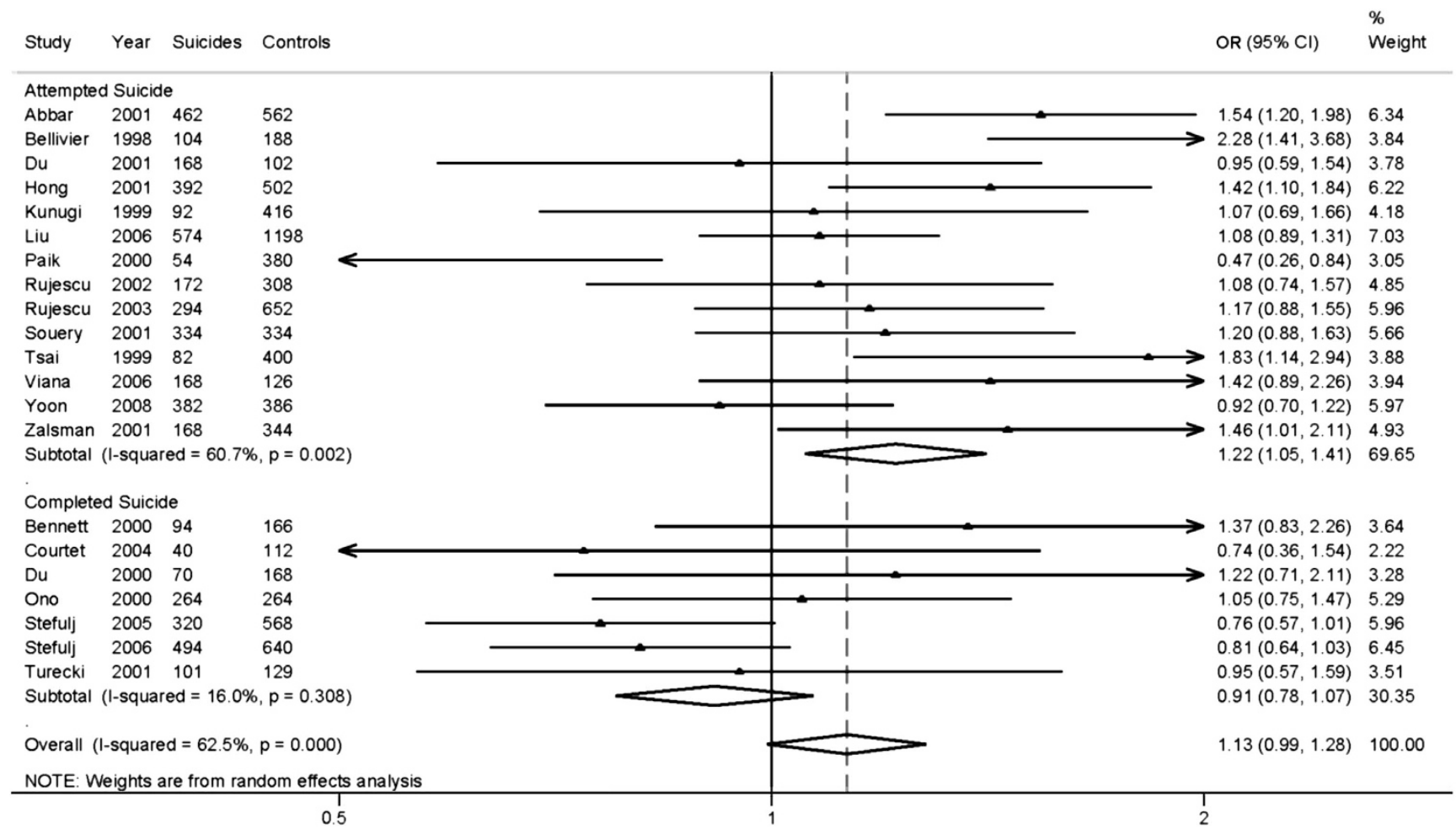

Figure 5 Forest plot for random-effects meta-analysis of the studies relating the tryptophan hydroxylase rs1800532 (A218C) variant to suicidal attempts, completions and overall suicidal behavior. We applied a random-effects inverse variance model to pool the odds ratios associating the TPH minor alleles with suicidal behavior. This plot describes the results for the 'A218' allele relative to the 'C218' allele.

Tryptophan hydroxylase. Multiple variants in the $T P H$ gene have been extensively explored in relation with suicidal behavior owing to the enzyme's role in serotonin metabolism. The two most studied SNPs, rs $1800532(n=21, \mathrm{NS}=4829$, $\mathrm{NC}=7945)$ and $\mathrm{rs} 1799913(n=8, \mathrm{NS}=1512, \mathrm{NC}=3408)$, revealed pooled ORs of $1.13(0.99-1.28), P=0.06$, power of $99 \%$ and $1.10(0.85-1.43), P=0.15$, power of $56 \%$. When the rs1800532 variant was selected for attempted suicides, the heterogeneity decreased slightly (from 62.5$60.7 \%$ ) and the pooled ORs showed a nominally significant harmful trend for the minor allele (1.22 (1.05-1.41), $P=0.007$, power of $99 \%$ ) (Figure 5). With the completed suicides, the heterogeneity decreased considerably (from 62.5-16.0\%); however, the pooled OR displayed a nonsignificant protective trend of association (0.91 (0.78-1.07), $P=0.31$, power of $47 \%$ ). 
COMT and MAOA. COMT and MAOA are two major enzymes involved in the metabolism of a variety of neurotransmitters. The rs4680 SNP of COMT $(n=9$, $\mathrm{NS}=3226, \quad \mathrm{NC}=3055)$ produced a pooled $\mathrm{OR}$ of 1.13 (0.89-1.42) $(P=0.32$, power of 93\%). Research regarding the two most common VNTR polymorphisms of MAOA (VNTR3 and VNTR4) produced five primary studies $(\mathrm{NS}=862, \mathrm{NC}=1239)$ and a pooled OR of $0.94(0.75$ 1.19) $(P=0.63$, power of $16 \%)$.

Brain-derived neurotropic factor. A recent meta-analysis by Zai et al. $^{26}$ indicated a strong association between the rs6265 SNP in the BDNF gene and suicidal behavior. In total, there were seven published studies $(N S=1700, N C=2584$ ) pertaining to the rs6265 SNP and suicidal behavior that met our inclusion criteria resulting in nonsignificant association (1.14 (0.83-1.56), $P=0.43$, power of $78 \%$ ) with suicidal behavior.

\section{Discussion}

In this study, we performed a systematic review and metaanalysis of all published studies investigating the association between genetic polymorphisms and suicidal behavior before August 2011. The serotonergic system has been extensively investigated in relation to suicidal behavior following early reports linking serotonin to suicide. ${ }^{27,28}$ Several of these related genes are involved in different pathways in serotonin production ( $T P H)$, transportation (SLC6A4), transmission (5HTR 1A, 1B, 2A and 2C) and metabolism (MAOA). For instance, SLC6A4 encodes the serotonin transporter protein that is expressed in platelets and a variety of neuronal membranes and is responsible for the uptake of serotonin into presynaptic neurons. ${ }^{29} \mathrm{~A}$ common polymorphism reported in 37 independent studies, known as the 5HTTLPR, is the result of a 44 base pair insertion (L) or deletion (S) in the promoter region of SLC6A4. ${ }^{12}$ The results of our random-effects metaanalysis indicate that the minor (S) allele in SLC6A4 increased the risk of suicide attempts by $13 \%(O R=1.13(1.05-1.21)$, $P=0.001)$ with low heterogeneity $\left(I^{2}=2.5 \%\right)$. In addition, the rs1800532 SNP of TPH, which encodes for the enzyme that catalyzes the formation of serotonin, was also nominally significantly related to suicidal attempts (1.22 (1.05-1.41), $P=0.007$ ) supporting previous research by Li et al. ${ }^{30}$ The four most commonly reported 5 HTR SNPs linking the $1 \mathrm{~A}, 1 \mathrm{~B}, 2 \mathrm{~A}$ and $2 \mathrm{C}$ receptor subtypes to suicidal behavior (rs6295, rs6296, rs6313 and rs6318) produced pooled ORs that were not statistically significant. Finally, the MAOA (VNTR 3/4), $B D N F$ (rs6265) and COMT (rs4680) polymorphisms also reported no significant association with suicidal behavior. Other non-serotonergic genes that have only recently been studied have demonstrated promising results in literature, including $S C N 8 A, D R D 2, A C E$ and $C C K^{31-34}$ All of these genetic variants will benefit from future research with larger sample populations.

After completing a systematic search for all published studies relating to the 35 genetic markers, we found 10 genetic variants that have been extensively studied in suicide (5HTTLPR, rs6295, rs6296, rs6313, rs6318, rs1800532, rs1799913, VNTR3/4, rs4680 and rs6265). Our modified Newcastle-Ottawa Rating Scale helped determine the quality of each study to narrow the inclusion of high-quality independent studies in our meta-analyses (Supplementary Information S2). From the 112 studies that remained, we derived pooled ORs with $95 \%$ Cls using the random effects DerSimonian and Laird inverse variance method. ${ }^{25}$ There was initially no association with the broad suicidal behavior trait for any variant reported in the literature; however, the degree of between-study heterogeneity was extremely high in most cases. Our subgroup analysis hypothesis stemmed from the small number of whole-genome linkage studies that indentified chromosomal regions of linkage for suicide attempts and that were not shared for fatal suicides. Theoretically, if the suicidal behavior continuum is driven by an increasing load of genetic markers, then fatal attempts (completions) and nonfatal attempt may exhibit a high level of redundancy in the context of linkage studies. However, this was not the case. Chromosomes 2p, 5q, 6q, 8p, $11 q$ and $X q$, with varied psychiatric comorbidities, were linked to nonfatal attempters whereas suicide completions identified only one linkage (MLOD > 2.50, $P<0.05$ ) at chromosome 6q..$^{9,35,36}$ As a result of this preliminary evidence, we chose a priori to undertake subgroup analyses by separating studies that included suicide attempts from suicide completions.

A true statistical independence between the two subgroups was not observed with these variants $\left(\chi^{2}>20, P<0.01\right)$ implicating a limited power in most suicide completion and two suicide attempt subgroups. However, the heterogeneity decreased significantly, in some cases by over $80 \%$, and two subgroup analyses (SLC6A4 5HTTLPR and TPH rs1800532) became at least nominally significant. Under conditions of high between-study heterogeneity, true associations with genetic variants may not be practically possible to replicate with consistency, no matter how many large studies are conducted. ${ }^{37}$ In part, the systematic pooling of these two distinct phenotypic subgroups may explain why no common variant contributing to suicide risk has been identified thus far. The results of these meta-analyses support this theory, and by separating suicide attempts from completions, we were able to identify genetic variants associated with suicide attempt, which will benefit from future research.

Despite the large number of studies included in our review, certain limitations are important to consider when interpreting our analyses. For instance, there was a lack of accessibility to studies dealing with suicidal behavior in individuals without psychiatric comorbidities. Only one study included a suicidal population without any comorbid psychiatric illnesses. As a result, a large majority of the studies we investigated involved suicidal behavior with varying psychiatric comorbidities that may contribute to the heterogeneity of the sample populations. In our systematic review of the literature, we were surprised by the number of genetic markers that showed nonsignificant results and a modest number of studies $(n<10)$. This low density of candidate gene studies in the suicide field may have severe consequences on the statistical power and the conclusiveness of some of our smaller metaanalyses. The $5 H T R 1 B$ rs6296 polymorphism, as an example, had a low power of $44 \%$ and a sample size of 2948 (compared with the SLC6A4 5HTTLPR polymorphism with a power of $99 \%$ and sample size of 6324 ), which may have contributed to the inconclusiveness of the pooled effect. It is 
also possible that an accumulation of smaller studies had a large effect on driving pooled significance toward a falsepositive result. Future studies should aim to examine these genes with larger sample size and adjusting for varying psychiatric comorbidities to increase the statistical power of results. Finally, future research should focus on larger population-based cases with matched controls as it is difficult to draw conclusions from small studies with a large number of confounding variables (including ethnicity, age, comorbidities and sex).

This study indicates that suicide attempts and completions should be considered as two distinct phenotypes and confirms that the reasons of between-study variation need to be carefully addressed in meta-analyses to produce unbiased conclusions. Although suicidal behavior may be a complex phenomenon resulting from the interplay of several genes, proteins, metabolites, environmental factors and psychiatric disorders, this paper has helped identify the significant genetic variants in hopes of finding those with a true association with suicidal behavior. Regardless of the limitations of the current literature, the results of our meta-analyses suggest that there are significant associations between the polymorphisms in the SLC6A4 and TPHgenes and suicide attempts. The increasing amount of effort spent on genome-wide studies will help improve our understanding of the genetic architecture of suicidal behavior, an important pre-requisite to better target individual risk factors for proper intervention and aid the at-risk population in managing the manifestation of suicidal behavior.

\section{Conflict of interest}

The authors declare no conflict of interest.

Acknowledgements. RCC and AZ received PHRI summer studentships for data extraction. DM is funded by a Tier 2 Canada Research Chair.

1. Mann JJ. Neurobiology of suicidal behaviour. Nat Rev 2003; 4: 819-828.

2. Sher $\mathrm{L}$. Brain-derived neurotrophic factor and suicidal behavior. QJM 104: 455-458.

3. Stoff DM, Mann JJ. Suicide research. Overview and introduction. Ann N Y Acad Sci 1997; 836: 1-11.

4. Roy A, Segal NL. Suicidal behavior in twins: a replication. J Affect Disord 2001; 66: 71-74.

5. Turecki G. Suicidal behavior: is there a genetic predisposition? Bipolar Disord 2001; 3: 335-349.

6. Voracek M, Loibl LM. Genetics of suicide: a systematic review of twin studies. Wien Klin Wochenschr 2007; 119: 463-475

7. Willour VL, Seifuddin F, Mahon PB, Jancic D, Pirooznia M, Steele J et al. A genome-wide association study of attempted suicide. Mol Psychiatry 2012; 17: 433-444.

8. Gibson G. Rare and common variants: twenty arguments. Nat Rev Genet 2012; 13 : 135-145.

9. Brezo J, Klempan T, Turecki G. The genetics of suicide: a critical review of molecular studies. Psychiatr Clin North Am 2008; 31: 179-203.

10. Arango V, Huang YY, Underwood MD, Mann JJ. Genetics of the serotonergic system in suicidal behavior. J Psychiatr Res 2003; 37: 375-386.

11. Fitch D, Lesage A, Seguin M, Trousignant M, Bankelfat C, Rouleau GA et al. Suicide and the serotonin transporter gene. Mol Psychiatry 2001; 6: 127-128.

12. Lesch K-P, Bengel D, Heils A, Sabol SZ, Greenberg BD, Petri S et al. Association of anxiety-related traits with a polymorphism in the serotonin transporter gene regulatory region. Science 1996; 274: 1527-1531.

13. Wang $S$, Zhang K, Xu Y, Sun N, Shen $Y, X u Q$. An association study of the serotonin transporter and receptor genes with the suicidal ideation of major depression in a Chinese Han population. Psychiatry Res 2009; 170: 204-207.
14. Ono $\mathrm{H}$, Shirakawa $\mathrm{O}$, Nishiguchi $\mathrm{N}$, Nishimura $\mathrm{A}$, Nushida $\mathrm{H}$, Ueno $\mathrm{Y}$ et al. No evidence of an association between a functional monoamine oxidase a gene polymorphism and completed suicides. Am J Med Genet 2002; 114: 340-342.

15. Lalovic A, Turecki G. Meta-analysis of the association between tryptophan hydroxylase and suicidal behavior. Am J Med Genet 2002; 114: 533-540.

16. Bellivier F, Leboyer M, Courtet $P$, Buresi $C$, Beaufils B, Samolyk D et al. Association between the tryptophan hydroxylase gene and manic-depressive Illness. Arch Gen Psychiatry 1998; 55: 33-37.

17. Zarrilli F, Angiolillo A, Castaldo G, Chiariotti L, Keller S, Sacchetti S et al. Brain derived neurotrophic factor (BDNF) genetic polymorphism (Val66Met) in suicide: a study of 512 cases. Am J Med Genet B 2009; 150B: 599-600.

18. Finckh U, Rommelspacher H, Kuhn S, Dufeu P, Otto G, Heinz A et al. Influence of the dopamine D2 receptor (DRD2) genotype on neuroadaptive effects of alcohol and the clinical outcome of alcoholism. Pharmacogenetics 1997; 7: 271-281.

19. Persson M-L, Geijer T, Wasserman D, Rockah R, Frisch A, Michaelovsky E et al. Lack of association between suicide attempt and a polymorphism at the dopamine receptor D4 locus. Psychiatr Genet 1999; 9: 97-100.

20. Stroup DF, Berlin JA, Morton SC, Olkin I, Williamson GD, Rennie D et al. Meta-analysis of observational studies in epidemiology: a proposal for reporting. Meta-analysis Of Observational Studies in Epidemiology (MOOSE) group. JAMA 2000; 283: 2008-2012.

21. Du L, Bakish D, Lapierre YD, Ravindran AV, Hrdina PD. Association of polymorphism of serotonin $2 \mathrm{~A}$ receptor gene with suicidal ideation in major depressive disorder. Am J Med Genet 2000; 96: 56-60.

22. Sequeira A, Mamdani F, Lalovic A, Anguelova M, Lesage A, Seguin M et al. Alpha 2A adrenergic receptor gene and suicide. Psychiat Res 2004; 125: 87-93.

23. American Psychiatric Association Diagnostic and Statistical Manual of Mental Disorders. DSM-IV-TR EditionNew YorkAmerican Psychiatric Association, 2000.

24. Gauderman WJ. Sample size requirements for matched case-control studies of gene-environment interaction. Stat Med 2002; 21: 35-50.

25. DerSimonian R, Laird N. Meta-analysis in clinical trials. Control Clin Trials 1986; 7 : $177-188$.

26. Zai CC, Manchia M, De Luca V, Tiwari AK, Chowdhury NI, Zai GC et al. The brain-derived neurotrophic factor gene in suicidal behaviour: a meta-analysis. Int $J$ Neuropsychopharmacol 2011 FirstView 1-6.

27. Stanley M, Virgilio J, Gershon S. Tritiated imipramine binding sites are decreased in the frontal cortex of suicides. Science 1982; 216: 1337-1339.

28. Stanley M, Mann JJ. Increased serotonin-2 binding sites in frontal cortex of suicide victims. Lancet 1983; 321: 214-216.

29. Langer SZ, Galzin AM. Studies on the serotonin transporter in platelets. Cell Mol Life Sci 1988; 44: 127-130.

30. Li D, He L. Further clarification of the contribution of the tryptophan hydroxylase (TPH) gene to suicidal behavior using systematic allelic and genotypic meta-analyses. Hum Genet 2006; 119: 233-240.

31. Wang Y, Zhang J, Liu B, Shao L, Wei Z, Li X et al. Genetic polymorphisms in the SCN8A gene are associated with suicidal behavior in psychiatric disorders in the Chinese population. World J Biol Psychiatry 2010; 11: 956-963.

32. Johann M, Putzhammer A, Eichhammer P, Wodarz N. Association of the $-141 \mathrm{C}$ Del variant of the dopamine D2 receptor (DRD2) with positive family history and suicidality in German alcoholics. Am J Med Genet B, Neuropsychiatric Genet 2005; 132B: 46-49.

33. Sparks DL, Hunsaker JC 3rd, Amouyel P, Malafosse A, Bellivier F, Leboyer M et al. Angiotensin l-converting enzyme I/D polymorphism and suicidal behaviors. Am J Med Genet B, Neuropsychiatric Genet 2009; 150B: 290-294.

34. Shindo S, Yoshioka N. Polymorphisms of the cholecystokinin gene promoter region in suicide victims in Japan. Forensic Sci Int 2005; 150: 85-90.

35. Cheng R, Juo SH, Loth JE, Nee J, lossifov I, Blumenthal R et al. Genome-wide linkage scan in a large bipolar disorder sample from the National Institute of Mental Health genetics initiative suggests putative loci for bipolar disorder, psychosis, suicide, and panic disorder. Mol Psychiatry 2006; 11: 252-260.

36. Willour VL, Zandi PP, Badner JA, Steele J, Miao K, Lopez V et al. Attempted suicide in bipolar disorder pedigrees: evidence for linkage to 2p12. Biol Psychiatry 2007; 61: 725-727.

37. Moonesinghe R, Khoury MJ, Liu T, Ioannidis JP. Required sample size and nonreplicability thresholds for heterogeneous genetic associations. Proc Natl Acad Sci USA 2008; 105: $617-622$.

Translational Psychiatry is an open-access journal published by Nature Publishing Group. This work is licensed under the Creative Commons Attribution-NonCommercial-No Derivative Works 3.0 Unported License. To view a copy of this license, visit http://creativecommons.org/licenses/by-nc-nd/3.0/ 